\title{
CIBO, SALUTE, PRETESE: RIFLESSIONI DALLA TEORIA DEI SISTEMI
}

\author{
FOOD, HEALTH, CLAIMS: INSIGHTS FROM SYSTEM THEORY
}

\author{
Sandra Regina Martini \\ Graduação em Ciências Sociais pela Universidade do Vale do Rio dos Sinos, \\ Mestrado em Educação pela Pontifícia Universidade Católica do Rio Grande do Sul, \\ Doutorado em Evoluzione dei Sistemi Giuridici e Nuovi Diritti pela Università Degli Studi di Lecce, \\ Pós-doutorado em Direito (Roma Tre) e Pós-doutorado em Políticas Públicas (Universidade de Salerno). \\ Pesquisadora Produtividade 2 - CNPq, Coordenadora do Mestrado em Direitos Humanos e \\ Professora do Centro Universitário Ritter dos Reis (Uniritter), \\ Professora-visitante no programa de pós-graduação em Direito da UFRGS (PPGD) \\ E-mail: srmartini@terra.com.br
}

Matteo Finco

Possui graduação em Scienze della Comunicazione e em Sociologia della Multiculturalità - Università degli Studi di Urbino, e doutorado em SOCIAL SCIENCES - System theory and sociology of normative and cultural processes - Università degli Studi di Macerata. Jornalista (registro de jornalistas profissionais italianos). Atualmente beneficia de uma bolsa de pesquisa de pós-doutorado CAPES no Centro Universitário Ritter dos Reis. Tem experiência na área de Sociologia, com ênfase em Teoria Sociologica.

Email: fincomatteo@gmail.com

CONVIDADOS

ABSTRACT: Scopo di questo lavoro è fornire un quadro di riferimento teorico per l'analisi di quelle che potrebbero essere definite le crescenti rivendicazioni di diritti e manifestazioni di interesse (individuali e collettive) relative al cibo nella contemporaneità. L'ipotesi, indagata attraverso un'analisi bibliografica nel contesto della teoria dei sistemi sociali di N. Luhmann, è che il cibo - e in particolare la sua tematizzazione nell'ambito della salute e del benessere - costituisca un mezzo attraverso cui gli individui possono avanzare pretese, costruendo la propria identità e attribuendo senso alle proprie condotte, trovando così una posizione all'interno del contesto sociale.

Parole chiave: Cibo. Salute. Teoria dei Sistemi Sociali.

ABSTRACT: This work aims to provide a theoretical framework for the analysis of those that nowadays could be defined as the growing claims of rights and manifestations of interest (individual and collective) related to food. The hypothesis, investigated through a bibliographic analysis in the context of N. Luhmann's social systems theory, is that food - and in particular its thematization in the field of health and well-being - constitutes a means that lets individuals can make claims, build their own identity and give meaning to their conduct, thus finding a position within the social context.

Keywords: Food. Health. Social System Theory. 
SOMMARIO: Introduzione; 1 Teoria dei Sistemi Sociali; 2 Salute/Benessere, Cibo e Inflazione di pretese; 3 Il cibo e i suoi “codici”; 4 Cibo come Diritto; 5 Cibo, Rischio, Salutismo; Conclusione; Riferimenti.

\section{INTRODUZIONE}

Negli ultimi decenni il cibo sembra aver assunto una posizione di primo piano a più livelli della panorama socio-culturale contemporaneo: nei media (con una overdose di programmi televisivi dedicati alla cucina, per non parlare della miriade di siti web che trattano di cibo e nutrizione in generale), nel mercato (dove crescono in parallello la domanda e l'offerta di prodotti diversificati, dalle origini più varie e dalle caratteristiche più eterogenee), nel diritto (con rivendicazioni crescenti dei cittadini-consumatori, sia in termini quantitativi che qualitativi: diritto al cibo quale diritto umano; importanza di un cibo salutare; diritti dei lavoratori che lo producono - "cibo etico"; ecc.).

Questi elementi (ma la lista potrebbe essere ben più lunga) stimolano a interrogarsi sull'origine di tanta attenzione: se in prima battuta verrebbe da dire che un maggiore benessere spinge a maggiori e più ampie pretese, non si può tuttavia fare a meno di pensare a una risposta più articolata.

L'ipotesi qui presa in considerazione - indagata a partire da un'analisi bibliografica nell'ambito della teoria dei sistemi sociali di N. Luhmann e articolata attraverso considerazioni di stampo prettamente teorico - è che il cibo - e soprattutto la sua tematizzazione nell'ambito della salute e del benessere - costituisca un mezzo attraverso cui diventa possibile dare efficacemente forma alla propria individualità. In altre parole, il cibo viene qui considerato un medium attraverso cui diventano possibili meccanismi di soggettivazione: non soltanto semplicemente mangiando, ma anche interrogandosi sul cibo (sue origini, produzione, caratteristiche) e soprattutto rivendicando diritti e pretese, gli individui possono contribuire a dare forma alla propria identità, in maniera personale e specifica. In questo modo la "ricerca di senso" trova un "oggetto" cui ancorarsi e diventa anche possibile relazionarsi in maniera più varia nel contesto sociale.

Nei termini della teoria dei sistemi, si tratta qui di lavorare sulla semantica del cibo - cioè sull'insieme dei temi ad esso relativi che alimentano (mai come in questo caso il gioco di parole sembra appropriato) la comunicazione all'interno della società nel suo complesso.

La scelta di concentrarsi in particolare sul collegamento fra cibo e salute/benessere è motivata dalla semplice constatazione che in quest'ultimo ambito le rivendicazioni di diritti e le manifestazioni di interesse (individuali e collettive) non solo appaiono crescenti, ma soprattutto risulta altamente improbabile la loro soddisfazione, per ragioni economiche (risorse scarse, specie nell'ambito delle politiche da realizzare), culturali (in un mondo sempre più globalizzato le differenze si fanno più evidenti e i conflitti più urgenti), sociali (si pensi solo alla complessità del diritto, con ordinamenti giuridici sempre più articolati).

\section{TEORIA DEI SISTEMI SOCIALI}

La teoria dei sistemi sociali elaborata nella seconda metà del Novecento dal sociologo tedesco Niklas Luhmann è nota per la sua complessità: ciò esclude la possibilità di fare più di qualche rapido ed essenziale cenno ai concetti utilizzati nel resto della presente trattazione.

Con la distinzione fra sistema e ambiente si indica, da un lato, l'insieme di elementi, in relazione tra loro, che contraddistingue un determinato ambito della società, il quale si riproduce in maniera continua a partire dalle proprie operazioni (comunicazioni) specifiche e che svolge, 
all'interno della stessa, una funzione anch'essa specifica (esempi: il diritto, la politica, l'economia); dall'altro, tutto ciò che non rientra nel sistema, essendo al suo esterno (anche gli esseri umani, per esempio).

La società moderna ( $\mathrm{si}$ badi bene, non postmoderna ${ }^{1}$ ) è una società-mondo (Weltgesellschaft ${ }^{2}$ complessa, policentrica, policontesturale, strutturata in base a una differenziazione di tipo funzionale (ogni sistema risolve, come detto, un problema diverso).

Gli esseri umani vengono inclusi nei sistemi (sociali) nella forma ${ }^{3}$ di persone, ovvero riferimenti per l'interazione e la comunicazione: entità in grado di comunicare ${ }^{4}$. L'individualità è allora possibile solo nella società moderna: è nella partecipazione ai differenti contesti della comunicazione (sistemi) che il singolo diviene individuo, cioè unico, differente da tutti i suoi simili e in grado di esercitare pretese (in particolare nella forma di diritti) ${ }^{5}$. L'identità individuale prodotto della continua ricerca di senso e continuativamente ridefinita - è il risultato - moderno di un processo fatto di decisioni (scelte e azioni) orientate da pretese (appunto di inclusione nei vari sistemi di funzione) ${ }^{6}$.

\section{SALUTE/BENESSERE, CIBO E INFLAZIONE DI PRETESE}

La salute non acquisisce, nell'intendimento di Luhmann, lo status di sottosistema di funzione della società: il sistema della medicina interviene su corpi e coscienze per renderli persone, cioè "adatti" alla comunicazione (si tratti semplicemente di ristabilire la piena funzionalità di un organismo o di far tornare un individuo al lavoro) ${ }^{7}$. La discussione sulla possibilità di considerare la salute un sistema di funzione a sé stante è in corso $^{8}$. Al di là di questo aspetto, la

\footnotetext{
1 Su questo si veda, ad esempio, LUHMANN, Niklas. Die Gesellschaft der Gesellschaft. Frankfurt am Main: Suhrkamp, 1997a.

${ }^{2}$ Cfr., fra gli altri, LUHMANN, Niklas. Globalization or World society: How to conceive of modern society? International Review of Sociology, Mar 1997b.

${ }^{3}$ Su questo concetto, cfr. SPENCER-BROWN, George. Laws of form. Bohmeier Verlag: Leipzig, 2014 (1969).

${ }^{4}$ Non comunicano infatti né i corpi (organismi viventi: sistemi biologici), né le coscienze (che riproducono pensieri: sistemi psichici).

5 Tale concetto verrà illustrato nel prossimo paragrafo.

${ }^{6}$ Ciò non era possibile nelle forme societarie precedenti, strutturate ad esempio per clan o ceti, in cui l'appartenenza a uno specifico contesto era determinata fin dal nascita e difficilmente poteva mutare. Sul rapporto tra individualità e modernità, cfr. in particolare: LUHMANN, Niklas. Individuo, Individualidad Suhrkamp, 1997a.

${ }^{6}$ Cfr., fra gli altri, LUHMANN, Niklas. Globalization or World society: How to conceive of modern society? International Review of Sociology, Mar 1997b.

${ }^{6} \mathrm{Su}$ questo concetto, cfr. SPENCER-BROWN, George. Laws of form. Bohmeier Verlag: Leipzig, 2014 (1969).

${ }^{6}$ Non comunicano infatti né i corpi (organismi viventi: sistemi biologici), né le coscienze (che riproducono pensieri: sistemi psichici).

${ }^{6}$ Tale concetto verrà illustrato nel prossimo paragrafo.

${ }^{6}$ Ciò non era possibile nelle forme societarie precedenti, strutturate ad esempio per clan o ceti, in cui l'appartenenza a uno specifico contesto era determinata fin dal nascita e difficilmente poteva mutare. Sul processo di «individualizzazione» quale dissoluzione delle forme di vita sociale precostituite, si veda anzitutto BECK, Ulrich. L'individualizzazione nelle società moderne. In: U. BECK, Ulrich; BECK-GERNSHEIM, Elisabeth (Org.). I rischi della libertà. L'individuo nell'epoca della globalizzazione. Bologna: il Mulino, 2000a, pp. 10-39).

${ }^{7}$ Luhmann parla di "Medizinsystem" ("sistema della medicina") e "System der Krankenbehandlung" ("sistema di cura delle malattie"). Cfr. LUHMANN, Niklas. Il codice della medicina (2015a). In: CORSI, Giancarlo (Org.). Salute e malattia nella teoria dei sistemi. A partire da Niklas Luhmann. Franco Angeli, Milano, 2015, pp. 40-51.

${ }^{8}$ Cfr., ad esempio, MARTINI, Sandra Regina. O sistema social da saúde: conceito, limites e possibilidades. Cad. IberoAmer. Dir. Sanit., 3, 1, (2014a), pp. 68-83.
} 
salute qui interessa quale ambito in cui diventa possibile, anzitutto attraverso pretese, la costruzione delle identità personali.

La prima difficoltà - evidente già soltanto a partire dalla definizione dell'Organizzazione mondiale della sanità ${ }^{9}$ - consiste nella vaghezza dell'idea di salute e nella sua continuità con il concetto di benessere. Basti questo per essere d'accordo sulla necessità di dover costruire continuamente (e ciò naturalmente nella comunicazione) tali idee ${ }^{10}$.

Tale continua ridefinizione - necessaria anche a causa dei continui progressi in campo scientifico e tecnologico e di tendenze alla "patologizzazione"/medicalizzazione - motiva gli individui a manifestare aspettative e pretese in tale ambito; aspettative che risultano crescenti: più tutele, più farmaci (e più economici), prestazioni differenziate ed efficaci, diritti sempre più specifici (come quelli "del malato" o come il "diritto alla dignità" o alla "buona morte". Si tratta, in breve, di quella che possiamo definire, con Luhmann, inflazione di pretese

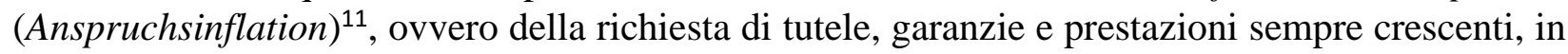
termini quantitativi (più diritti, per tutti) e qualitativi (standard più elevati): pare sempre più difficile dunque stabilire un limite a ciò che può essere rivendicato, anzitutto nell'ambito dei diritti (e in particolare di quelli soggettivi) ${ }^{12}$. Le pretese costituiscono dunque una caratteristica della società moderna, in quanto rappresentano un correlato della differenziazione funzionale: l'individuo-persona, venendo meno la possibilità di trovare dei punti di riferimento e riconoscersi in identità collettive forti, può autoidentificarsi solamente "in base a pretese e connettersi all'esperienza basata sulla loro soddisfazione o delusione"13.

Poiché la salute rappresenta "un valore di rilevanza massima e indiscutibile", l'unico "in grado di collocarsi al di là di qualsiasi controversia ideologica"14, è dunque possibile interrogarsi sull'importanza e sulle caratteristiche specifiche delle rivendicazioni relative a un ambito ancor più specifico a esso interno: il cibo. Ciò risulta particolarmente interessante in una società "sempre più globale" in cui (con i dovuti distinguo) il cibo rappresenta una risorsa largamente disponibile in forme sempre più varie.

\footnotetext{
e Id., Construção do sistema social da saúde a partir da Teoria Sistêmica de Niklas Luhmann. Revista de Direito sanitario, 16, 1, 2015, pp. 112-127.

${ }^{9}$ Che definisce la salute "uno stato di completo benessere, fisico, mentale e sociale, e non soltanto l'assenza di malattie e infermità": WHO. Constitution of the World Health Organization. 1946.

${ }^{10}$ Occorre cioè tutto un insieme di idee, procedure, figure e aspettative "alle quali ci si possa orientare per capire di cosa si sta parlando": CORSI, Giancarlo. Introduzione. Malattia e salute: il contributo della teoria dei sistemi. In: Id. (Org.). Salute e malattia nella teoria dei sistemi. A partire da Niklas Luhmann. Franco Angeli, Milano, 2015. p. 23.

11 Da ultimo, "Può così costituirsi una sorta di super-diritto soggettivo, un diritto ai diritti, vale a dire un diritto al continuo ampliamento delle pretese individuali”: LONGO, M. Strutture di società e semantica del soggetto. Lecce: Pensa Multimedia Editore, 2001. p. 290.

${ }^{12}$ In questo senso Luhmann parla di una semantica incrementale: "I sistemi prescrivono a se stessi una semantica basata sulla crescita illimitata": LUHMANN, Niklas. Inflazione di pretese nel sistema delle malattie: una presa di posizione dal punto di vista della teoria della società (2015b). In: CORSI, Giancarlo (Org.). Salute e malattia nella teoria dei sistemi. A partire da Niklas Luhmann. Franco Angeli, Milano, 2015, pp. 52-70. p. 53.

${ }^{13}$ Ibidem, p. 57.

${ }^{14}$ Ibidem, p. 62.
} 


\section{IL CIBO E I SUOI “CODICI”}

Nella teoria dei sistemi sociali ciascun sistema opera in base a un codice specifico ${ }^{15}$, cioè una distinzione che orienta la comunicazione, fornendo, in parole povere, un riferimento per capire cosa fare dopo: attraverso il codice (cioè una distinzione) il sistema osserva le proprie operazioni e tratta ogni possibile oggetto ${ }^{16}$.

Ora, rimanendo qui ben distanti anche soltanto dall'ipotesi di considerare il cibo come sistema di funzione, sembra possibile utilizzare il concetto di codice quale "chiave" in grado di attivare la comunicazione relativamente al cibo: in questo modo, diventa possibile distinguere e analizzare le molteplici dimensioni in cui il cibo diviene "oggetto" (tema) di comunicazione e, di conseguenza, di pretese.

Anzitutto osserviamo che il cibo viene "concepito" in maniera differente nei diversi sottosistemi della società: ad esempio è una merce dal punto di vista dell'economia (riguarda gli scambi commerciali, il consumo, il lavoro); negli ambiti giuridico ed etico viene inteso generalmente come diritto fondamentale, come bene dell'umanità a cui tutti dovrebbero avere ampio e facile accesso; è un prodotto culturale (parliamo di tradizioni culinarie, storia ed identità locali); un tema di comunicazione per i media (basti pensare all'alto numero di trasmissioni televisive e di siti web dedicati alla gastronomia) e costituisce sui social network un tema iperinflazionato (si pensi a fenomeni quali il food porn su piattaforme come Instagram e Facebook ${ }^{17}$ ); è al centro degli interessi della scienza e della tecnologia (sia in quanto alimento - si è addirittura arrivati a produrre finta carne in laboratorio, ovviando alla necessità di uccidere animali - sia per gli effetti che la produzione, la raccolta e la distribuzione del cibo hanno sull'ecosistema).

Il fatto di assumere in ogni sistema una forma differente, porta il cibo al centro di conflitti fra i sistemi stessi: conflitti dettati proprio dalla specificità dei sistemi. Se ad esempio il "diritto fondamentale al cibo" trova un ostacolo pressoché insormontabile dal punto di vista economico (le risorse sono scarse per definizione), l'ampliamento del mercato a livello mondiale influenza le possibilità di scelta degli individui, e di conseguenza le loro abitudini. Non mancano poi le problematiche ecologiche: spreco degli alimenti non consumati, inquinamento e sfruttamento del territorio dovuti a produzione, distribuzione, commercializzazione. Nell'ambito della salute (e del benessere) di individui e popolazioni, si incrociano poi varie dimensioni con la loro relativa problematicità. Anzitutto non si tratta soltanto di somministrare cure e trattamenti in caso di malattie, disturbi, disfunzioni, ma anche di prevenzione, di miglioramento di qualità della vita; di intervenire, insomma, in assenza di problemi conclamati: l'intervento è ormai la norma ${ }^{18}$. Inoltre, come detto, salute e cibo vengono rivendicati - in forme sempre più varie - quali diritti: si pensi non solo all'accesso alle risorse, ma anche alla sicurezza dei cibi, che impone controlli.

\footnotetext{
15 Talvolta più di uno, ma vi è sempre un codice primario: ad esempio il codice primario della salute è sano/malato, mentre geneticamente perfetto/geneticamente preoccupante e cubile/incurabile rappresentano codificazioni ulteriori.

${ }^{16}$ Cfr. BARALDI, Claudio; CORSI, Giancarlo; ESPOSITO, Elena. Luhmann in Glossario. I concetti fondamentali della teoria dei sistemi sociali. Milano: Franco Angeli, 2002. p. 63.

${ }^{17}$ Cfr. Marrone, Gianfranco. Food porn: l'esposizione del cibo. In: MASSIDDA, Luca (Org.). EXPO 1851-2015. Storie e immagini delle Grandi Esposizioni. Torino: Utet, 2015b, pp. 3-6.

18 "Si potrebbe quindi immaginare che il sistema intervenga solo se qualcuno si ammala. Ma questo non è vero (o non lo è più).": LUHMANN, Niklas. Il codice della medicina (2015a). In: CORSI, Giancarlo (Org.). Salute e malattia nella teoria dei sistemi. A partire da Niklas Luhmann. Franco Angeli, Milano, 2015, pp. 40-51. p. 46.
} 
Quest'ultimo aspetto si complica poi quando si vuole documentare l'origine dei prodotti per questioni economiche, culturali, identitarie (un prodotto tipico di un determinato territorio rappresenta una garanzia di qualità e ha per questo un valore economico più elevato di uno della grande distribuzione).

Tentiamo di seguito un primo elenco di codici relativi al cibo, utile sia per le riflessioni successive, sia in vista di lavori futuri.

Il cibo può essere inteso anzitutto in base al gusto: così il primo codice è buono/cattivo. Si rimane in questo senso a un livello basilare, ma comunque fondamentale, anche nell'ambito del mercato. Non si tratta solo di una dimensione "istintiva": ovviamente ricerchiamo ciò che è gradevole ed evitiamo ciò che non lo è. Ma la cultura gioca qui un ruolo decisivo: il gusto è una questione di appartenenza, di educazione e apprendimento ${ }^{19}$.

I media dal canto loro esaltano il fascino visivo degli alimenti: i reality show, i programmi televisivi, i siti web dedicati alla cucina, contribuiscono certamente anche a raccontare, rielaborare e trasmettere le tradizioni culinarie, le identità locali, e su tali elementi fanno leva per attrarre il loro pubblico; inoltre si reggono su elementi tradizionali nel mondo dell'intrattenimento e dello spettacolo (ad esempio puntando su personaggi già noti o su altri dall'elevato "potenziale mediatico", che raggiungono così notorietà); soprattutto però giocano sugli aspetti visivi: piatti dai colori sgargianti, "impiattamenti" raffinati, combinazioni improbabili di alimenti, ecc. In questo campo un codice potrebbe essere bello (attraente)/brutto. Altri possibili codici: raffinato-ricercato (straordinario)/comune (ordinario).

In ambito economico, la questione del prezzo dei cibi è essenziale: accessibile/non accessibile (o valore economico/elevato). Al di là di questo elemento c'è poi la questione della disponibilità dei beni (naturalmente proporzionale al prezzo): cibo ricercato/comune, ma anche distribuzione larga/ristretta, disponibilità ampia/ridotta.

Relativamente al diritto e all'etica del lavoro, un prodotto alimentare può essere "giusto" oppure no (etico/non etico). Se i diritti dei lavoratori non sono stati rispettati, se essi sono stati sfruttati o sottopagati, se insomma dal loro sudore sono stati tratti profitti indebiti, o ancora se la produzione ha tratto beneficio da meccanismi illeciti, una valutazione etica (negativa) diviene inevitabile.

In ambito ecologico - ambiente naturale, sostenibilità, energia - la distinzione principale potrebbe essere sostenibile/non sostenibile. Anche qui c'è una forte connotazione etica. Anche qui,

\footnotetext{
${ }^{19} \mathrm{Cfr}$. su questo, BOURDIEU, Pierre; Saint-Martin Monique. Goftts de classe et styles de vie. In: Anatomie du goftt. Actes de la Recherche en Sciences Sociales, 5, out. 1976, pp. 18-43. Dunque "non esiste il gusto in assoluto, ma esiste un gusto che si basa su una frequentazione, sulla permanenza in un contesto di paesaggi, aromi, eccessi e difetti, spezie ed evanescenze.”: LA CECLA, Franco. La pasta e la pizza. Bologna: il Mulino, 1998. Il gusto, inoltre, può essere inteso come un linguaggio e dunque come processo per l'attribuzione di senso: "taste is the language par excellence, the most silent and least studied. It is an actual primary cultural modelling system [...] in the field of taste the passage from perception to meaning is necessarily immediate. There is no taste without identification of value.": MARRONE, Gianfranco. Food meaning: From tasty to flavorful. Semiotica, 211, 2016, pp. 187-201. p. 2). Più in generale ancora, il cibo "costituisce una forma specifica di linguaggio. Di un cibo cioè, come ha sostenuto un antropologo fortemente interessato alla linguistica come Claude Lévi-Strauss (1962), "buono da pensare": attraverso il cibo parliamo del mondo, della società, del cosmo, di tutto insomma."; la cucina può anche essere considerata "come fatto sociale totale che tende a produrre forme di identità e di alterità etniche. I cibi sono segni, di noi stessi innanzitutto, del mondo sociale in secondo luogo, dell'universo intero infine.": MARRONE, Gianfranco. Senso e forma del cibo. Sulla semiotica dell'alimentazione. In: MONTANARI, Massimo (Org.). Il cibo nelle arti e nella cultura. Torino: Utet, 2015c, pp. 3-10. p. 4.
} 
inoltre, l'aspetto economico gioca un ruolo di primo piano: una tecnica agricola, un processo produttivo o di riciclaggio dei rifiuti, possono essere anch'essi sostenibili (convenienti) oppure no.

Dal punto di vista socio-culturale vanno considerate le dimensioni - di cui si è già parlato - relative a tradizioni, identità, territorio e più in generale tutto ciò che può andare sotto l'etichietta di "cultura" in senso lato. Riguardo in particolare l'aspetto identitario-comunitario del cibo, sono in gioco le tipicità locali e il legame dei prodotti con i territori e la loro storia. Pure qui è presente un intreccio con la dimensione economica (non si tratta soltanto di commercializzare i prodotti: si pensi ad esempio alle potenzialità, dal punto di vista turistico, di una determinatà località o di un territorio più o meno ampio). Le distinzioni possibili in tale ambito sono allora locale/globale $\mathrm{e}$ tradizione/novità. Le cose però si complicano se si considera che determinate tradizioni culinarie possono avere un successo che trascende i confini territoriali: come ha scritto J. Goody, "ingredienti, metodi di cottura e piatti regionali" vengono utilizzati da cuochi (e dai più raffinati chef) "che cucinano per gente la cui competenza, gusto e obiettivi trascendono una singola località" e che portano su una dimensione nazionale le cucine locali ${ }^{20}$. Si pensi poi alle rielaborazioni all'estero delle tradizioni culinarie: ad esempio ai ristoranti cinesi nel mondo, con menu molto differenti da quelli in patria; ai ristoranti giapponesi in Italia, la gran parte dei quali con personale di nazionalità cinese; o ancora alle cucine tex-mex e ai sushi-bar, ecc. ${ }^{21}$

Anche in questo ambito la tendenza alla moralizzazione è elevata: la tradizione (origine) viene connotata positivamente, mentre la novità è (quantomeno all'inizio) guardata con sospetto, quando non apertamente avversata.

Lo stesso, peraltro, vale con un'altra distizione: naturale (genuino)/artificiale. Qui siamo nel campo della scienza e della tecnica: i progressi in tale settore sono continui, e al di là del loro sfruttamento dal punto di vista commerciale, è innegabile l'importanza di nuove scoperte e tecnologie per garantire un accesso più ampio alle risorse (si torna nell'ambito dell'economia, del diritto, della morale e dell'etica). Problematico risulta però l'aspetto comunicativo: proliferano scetticismo e false convinzioni, con il successo di distinzioni senza fondamento scientifico (ad esempio si qualificano come "artificiali" prodotti definitivi spregiativamente e genericamente "chimici", e "naturali" vengono chiamati prodotti derivanti dall" "agricoltura biologica"22: ma l'agricoltura è di per sé "artificiale", essendo un insieme di tecniche di lavorazione e sfruttamento che l'umanità ha affinato nel corso del tempo).

Passando all'ambito che più ci interessa, quello della salute, l'elemento centrale è ovviamente la salubrità dei cibi (codice: salutare/non salutare o, in termini più comuni, cibo sano/cibo 'spazzatura'). Tuttavia, di fronte alla ricorrente proposizione dei cosiddetti "supercibi" e in generale di alimenti elogiati per le loro proprietà benefiche (talvolta eccezionali in quanto straordinamente "ricchi" di sostanze nutritive e di elementi quali fibre, antiossidanti, ecc., che

\footnotetext{
${ }^{20}$ GOODY, Jack. Cooking, Cuisine, and Class. A Study in Comparative Sociology. Cambridge: Cambridge University Press, 1982. p. 73.

${ }^{21}$ Riguardo l'aspetto identitario, va ricordato in generale che "Se, come si ripete, l'uomo è ciò che mangia, non è tanto o soltanto perché le sostanze che via via incorpora vanno a costituire la sua materialità fisica, quanto anche perché, dal punto di vista antropologico, il cibo che prepara e ingerisce lo rappresenta, lo significa, contribuendo a costruirne l'identità, individuale come collettiva." (MARRONE, op.cit. (2015c), p. 3). Sul rapporto fra cibo, cultura e identità, si veda inoltre DOUGLAS, Mary. Il cibo come sistema di comunicazione. In: Antropologia e simbolismo. Religione, cibo e denaro nella vita sociale. Bologna: il Mulino, 1985 ("il cibo serve a delimitare una identità in pericolo": p. 59). ${ }^{22} \mathrm{Si}$ veda su questo MARRONE, Gianfranco. La forma del latte. Allusioni erotiche ed emergenze del reale. Lexia. Rivista di semiotica, 19-20 giugno 2015a, pp. 331-346, che parla di una "ideologia naturalistica (il cosiddetto mondo del biologico o dell'organic) tanto diffusa fra la massa dei consumatori” (cit. p. 332).
} 
portano al rallentamento dell'invecchiamento o prevengono malattie - in particolare i tumori - o che magari, più banalmente, facilitano i normali processi biologici, e altro ancora), si può pensare appunto un codice cibo ordinario/straordinario ('super'). A questo livello agiscono insieme l'ambito dei media, quello della scienza e quello del commercio: vuoi per una voluta "complicità" o, più banalmente, a causa della scarsa competenza dei divulgatori. Particolarmente interessanti a questo proposito sono le svariate mode che si affermano con cadenza regolare: diete particolari come la "paleolitica", la cucina macrobiotica, e molte altre ancora, per non parlare del crescente successo del vegetarianesimo e del veganesimo ${ }^{23}$. Si tratta di fenomeni che testimoniano l'attribuzione al cibo di un particolare valore dal punto di vista della salute e del benessere: tale attribuzione avviene tuttavia secondo differenti modalità, non sempre trasparenti. Se è dalla scienza (che difatti nella teoria dei sistemi è caratterizzata dal codice vero/non vero) e dalla tecnica che ci si aspetterebbero risposte e giudizi sufficientemente sicuri, stabili e affidabili sulla salubrità dei cibi, è al contrario evidente che così non avviene. Ciò ha ricadute importanti nell'ambito delle pretese (in particolare: diritti) relative al cibo sano. È questo, dopotutto, l'elemento centrale del presente lavoro.

\section{CIBO COME DIRITTO}

"Ogni individuo ha diritto ad un tenore di vita sufficiente a garantire la salute e il benessere proprio e della sua famiglia, con particolare riguardo all'alimentazione"24: così recita 1'articolo 25 della Dichiarazione Universale dei Diritti dell'Uomo del 1948. Non si può negare che nel frattempo siano stati fatti passi avanti: si pensi ad esempio a documenti e regolamenti sulla sicurezza alimentare come il White Paper on Food Safety e la General Food Law Regulation (Regulation (EC) No 178/2002) adottata dal Parlamento e dal Consiglio europei, o alla creazione di organismi quali la European Food Safety Authority (EFSA) e il Rapid Alert System for Food and Feed (RASFF).

Il diritto a un'alimentazione sicura, qualitativamente e quantitativamente soddisfacente, costituisce una sfida per la società moderna e, di conseguenza, per i vari sistemi che "trattano" di cibo. C'è tuttavia il problema, già visto, dei conflitti tra sistemi; ma soprattutto, appare evidente la difficoltà del compito di trovare un equilibrio tra valori quali salute e sicurezza - rivendicati anzittutto dagli individui, sia in quanto esseri umani (si può allora parlare di "diritto umano al cibo"), sia in quanto cittadini/consumatori - e diritti rivendicati da vari settori, ambiti, organismi, istituzioni della società (come i principi del libero mercato e della concorrenza, o la libertà di impresa), che sono necessariamente parziali e soprattutto non facilmente compatibili con i diritti soggettivi degli individui.

Quando si parla di salute e benessere (e di conseguenza di nutrizione, sicurezza, ecc.) si parla di concetti, cioè idee, principi, ideali, valori: difficili da definire, e ancor di più da attuare, anche soltanto presi singolarmente. Figurarsi poi la difficoltà nello stabilire gerarchie. Ad esempio: come equilibrare la richiesta di elevati standard di sicurezza alimentare con quella di un apporto nutrizionale appropriato?

\footnotetext{
${ }^{23}$ Non si vuole qui certamente negare la peculiarità (e l'importanza) di tali regimi relativamente alla loro sostenibilità dal punto di vista ambientale. Più problematico, invece, il caso dell'agricoltura "biologica": qui la comunità scientifica non ha ancora raggiunto un giudizio condiviso.

${ }^{24}$ Assemblea Generale delle Nazioni Unite. Dichiarazione Universale dei Diritti dell’Uomo. 1948.
} 
In questo modo, pensare al cibo come diritto umano diventa quasi una pretesa insostenibile, almeno da un punto di vista scientifico: si tratta, ancora una volta, di valori ancor prima che di diritti. Di qui la sfida per il sistema politico, chiamato alla creazione di norme.

\section{CIBO, RISCHIO, SALUTISMO}

La difficoltà di soddisfare le pretese relative al cibo dovrebbe essere a questo punto sufficientemente chiara.

$\mathrm{Ci}$ sono ancora almeno due aspetti, tuttavia, che vale la pena tenere in debita considerazione trattando del legame fra cibo, salute e individualità: il primo è relativo al concetto di rischio; il secondo a una deriva dell'idea di salute, identificabile in quella che potrebbe essere definita "ideologia del salutismo". Analizziamo tali aspetti in sequenza.

Rispetto al rischio, la premessa è che se le pretese, di qualsiasi tipo, mirano a ottenere un vantaggio, un miglioramento, d'altra parte è vero che il rischio - di perdere qualcosa - è sempre presente. Inoltre, se il cibo e la salute sono ambiti tanto importanti per la costruzione dell'identità individuale, ciò significa che gli individui stessi, attraverso le proprie scelte e decisioni continue, non possono evitare di correre rischi.

Ma quali sono origine e caratteristiche più specifiche di tale concetto?

Su questo tema la sociologia ha lavorato negli ultimi decenni con attenzione costante e risultati apprezzabili, analizzandone in particolare la natura tipicamente moderna ${ }^{25}$. U. Beck, con l'espressione "società del rischio", ha evidenziato che si tratta di un prodotto della modernizzazione. Allo sviluppo di forze produttive si accompagna quello di pericoli distruttivi: sono la scienza e la tecnologia, in primo luogo, a generare potenziali pericoli e catastrofi; i rischi, poi, sono allo stesso tempo locali e globali, coinvolgendo uomini, animali e piante, e con possibili effetti nel mondo intero (si pensi ai pericoli nucleari).

Guardando poi all'etimologia del termine, occorre tornare al XVII secolo, con l'affermazione della convinzione relativa al fatto che "alcuni vantaggi non possano essere ottenuti se non si mette in gioco qualcosa" 26 . Progressivamente perde consistenza l'idea di sicurezza (securitas): si sa, e si accetta, che possano esserci imprevisti, dunque incertezza e insicurezza ${ }^{27}$.

Luhmann ha evidenziato l'importanza del concetto di decisione: attraverso esso si giunge alla distinzione moderna fra rischio e pericolo: danni e svantaggi sono sempre possibili, ma da una parte troviamo quelli derivanti da decisioni proprie (rischio), dall'altra quelli prodotti da fattori esterni sui quali non abbiamo controllo (pericolo), che sono dunque ineliminabili in quanto indipendenti dalle decisioni (banalmente: un albero può sempre cadere a causa di un fulmine). La decisione invece porta sempre qualche rischio (non rischierò di essere vittima di un fulmine se non decido di uscire di casa). Ciò a cui i rischi danno accesso (ed è questa la ragione per cui vengono corsi) è un possibile vantaggio. Di qui l'importanza della decisione: se voglio tentare di cogliere un'opportunità, devo rischiare, esponendomi a possibili insuccessi che possono determinare non

\footnotetext{
${ }^{25}$ Cfr. fra gli altri: BECK, Ulrich. Un mondo a rischio. Torino: Einaudi, 2003; Id. La società del rischio. Roma: Carocci editore, 2000b; Id. Dalla società industriale alla società del rischio. Questioni di sopravvivenza, struttura sociale e illuminismo ecologico. Teoria sociologica, 1, 1993, pp. 49-75; LUHMANN, Niklas. Society Shocked by Its Risks. Social Sciences Research Centre Occasional Paper, 17, 1996b; Id. Sociologia del rischio. Milano: Bruno Mondadori, 1996a (Soziologie des Risikos. Berlin: de Gruyter, 1991)..

${ }^{26}$ N. Luhmann, op. cit. (1996a), cit., p. 19.

${ }^{27}$ Se non lo si fa, d'altro canto, "non è che le catastrofi scompaiano, mentre l'agitazione produce dei danni che si potrebbero evitare": LUHMANN, Niklas. CEVOLINI, Alberto (Org.). Il rischio dell'assicurazione contro i pericoli. Roma: Armando, 2013.
} 
solo mancati miglioramenti, ma anche peggioramenti: gli orizzonti futuri, in questo senso, cambiano inevitabilmente ogni volta che si compie una decisione.

D'altronde non è possibile evitare di prendere decisioni: anche quella di "non decidere" è infatti una decisione, come quella di "non rischiare" (che appunto preclude l'accesso a vantaggi futuri al momento ignoti e imprevedibili).

Occorre poi considerare che $i$ rischi portano ad altri rischi: lo strumento dell'assicurazione ${ }^{28}$, d'altronde, serve proprio in vista di possibili circostanze sfavorevoli. Se però queste ultime non si verificano, si può pensare di aver pagato inutilmente. Inoltre le decisioni prese condizioneranno in maniera determinante ulteriori decisioni future, che genereranno a loro volta altri rischi. Anche per questo, parallelamente all'incremento delle conoscenze, emergono problemi in precedenza inimmaginabili.

I termini decisione, rischio e opportunità sembrano allora formare un consistente "blocco semantico" caratteristico della società-mondo, essendo strettamente legati a incertezza, complessità, determinazione del futuro.

Tornando al cibo e alla salute, va sottolineato che la distinzione fra rischio e pericolo sottintende quella fra decisori e coinvolti. In questo ambito ciò risulta particolarmente importante: lo si vede in particolare dalla scarsa disponibilità ad accettare esiti sfavorevoli indipendenti dalla propria volontà, diversamente da quanto accade con quelli derivanti da propri comportamenti consapevolmente scelti ${ }^{29}$.

Possiamo constatare più livelli in cui le scelte relative al cibo comportano rischi: alcuni non si possono immaginare, altri sono legati a certi tipi di alimenti (sicuramente carne e pesce portano più rischi rispetto alle verdure, a causa delle potenziali malattie degli animali), altri ancora dipendono da specifiche sostanze o componenti "notoriamente" rischiose (si pensi ai tanto demonizzati grassi) ${ }^{30}$.

L'estensione stessa dei concetti di salute e benessere porta a considerare le "virtù terapeutiche" degli alimenti, così che il cibo in generale viene progressivamente assimilato alla medicina, con il risultato che diventa sempre più difficile distinguere fra $\mathrm{i}$ due termini ${ }^{31}$.

Di conseguenza, perseguire la salute diviene un compito sempre più rischioso e difficile, ma anche per questo sempre più importante. Di qui il passaggio all'analisi del salutismo quale ideologia contemporanea. La nutrizione diviene cioè non solo un campo in cui viene modellata l'individualità, ma assume anche un valore in sé, diventando da ultimo un imperativo morale. Si tratta naturalmente di un approccio riduttivo rispetto a cibo e salute ${ }^{32}$, che sfocia facilmente in forme patologiche e nell'ambito della morale: come visto, si arriva a classificare a prescindere certi cibi come "buoni", "salutari", e altri come "cattivi". Non solo: gli individui stessi - a partire dagli stessi presupposti già illustrati dell'individualità contemporanea investita dall'obbligo di

\footnotetext{
${ }^{28}$ L'assicurazione ha le sue origini nel XVIII secolo, nell'ambito del commercio marittimo: cfr. CEVOLINI, Alberto, Presentazione. In: LUHMANN, Niklas; CEVOLINI, Alberto (Org.). Il rischio dell'assicurazione contro i pericoli. Roma: Armando, 2013.

29 Ad esempio: "Preferiamo morire per un'alimentazione sbagliata piuttosto che per gli effetti della chimica alimentare": LUHMANN, Niklas, op. cit. (2013), p. 58.

${ }^{30}$ Seguiamo qui la seguente classificazione: "i) food safety hazards, ii) nutrition-informed risks, and iii) risks of the unknown.”: SILCHENKO, Ksenia. Powered by Health: Healthism in Food Marketing and Consumer Research. A Systematic Revier and a Critical Disourse Analysis. 2017. Tese (Doutorado) - Università degli Studi di Macerata, 2017. p. 65.

${ }^{31}$ MAYES, Christopher. Medicalization of eating and feeding. In: THOMPSON Paul B.; KAPLAN, David M. (Orgs.). Encyclopedia of food and agricultural ethics. Dordrecht: Springer, 2014, pp. 1-8. p. 5.

${ }^{32}$ SILCHENKO, op. cit., p. 69.
} 
decidere - vengono connotati moralmente ("virtuosi" e non) a seconda dei loro comportamenti alimentari, e si autoresponsabilizzano, in maniera talvolta insostenibile ${ }^{33}$. La salute diventa, in altre parole, un dovere ${ }^{34}$.

Di fronte a tali fenomeni, la scienza sociologica potrebbe lavorare per tentare di prendere in considerazione insieme, in un unico quadro, tendenze opposte: gli aspetti ideologici, le mode, $\mathrm{i}$ condizionamenti biopolitici da un lato; ma anche, dall'altro, una maggiore consapevolezza individuale e la possibilità crescente di scelta ${ }^{35}$.

\section{CONCLUSIONE}

Se la moda delle diete (più svariate) può essere considerata un fenomeno tipicamente moderno, oggi più che mai, connesso all'ideologia del salutismo e all'inflazione di pretese, è pur vero che da sempre la "dietetica" ha rappresentanto un campo per la costruzione dell'individualità ${ }^{36}$. Una costruzione che avviene non soltanto privatamente, ma apertamente nel contesto sociale $^{37}$. Più in generale, non solo il cibo, ma la salute stessa, già al livello del senso comune, va ben oltre la medicina e costituisce un percorso codificato in cui si agisce tenendo come riferimento situazioni ideali, a livello individuale, sociale e $\operatorname{cosmico}^{38}$.

Stabilire cosa sia un salutare riguardo al cibo appare allora un compito arduo, ma soprattutto non predeterminato e che rimanda all'individuo. Per quanto la salute possa essere messa al centro delle politiche (locali, nazionali, internazionali) è sempre al singolo individuo-persona (e dunque anche soggetto di diritti) che spettano la formulazione e la ridefinizione continua di salute e benessere, di cosa sia salutare, insieme al compimento di scelte e azioni conseguenti. La possibilità di scegliere stessa espone, come visto, a rischi (tali proprio in quanto assunti consapevolemente, anche se non del tutto prevedibili): di qui, se da un lato tendono a mancare nella società orientamenti stabili e condivisi e se si rischia, fra le altre cose, un certo isolamento, dall'altro risulta aperta la strada ad una responsabilizzazione individuale che a sua volta rimanda alla presa di coscienza del proprio ruolo nel contesto sociale: le scelte individuali, dopottutto, hanno conseguenze a livello comunitario/societario.

Il cibo appare così un oggetto, uno strumento, un mezzo attraverso cui l'individualità prende letteralmente corpo (e davvero: non solo a livello figurato!). Un mezzo, un oggetto, a cui si continuerà sempre a far ricorso, perché si tratta di quanto più prossimo ed essenziale alla sopravvivenza stessa degli esseri umani. Sia al livello corporeo-organico, che a livello simbolico. Nulla come la salute - intesa anzitutto quale valore condiviso - trova infatti nel cibo un canale più

\footnotetext{
${ }^{33}$ Qui non ci inoltriamo in una lettura biopolitica della questione, che tuttavia appare senz'altro opportuna per un'analisi completa del fenomeno.

34 "in healthism choosing health food is a moral pleasure and hence also a duty. A growing ability to make healthy food choices translates into an immense moral responsibility consumers must take (if they can) in order to minimize treatment costs and to maximize the happiness of people around": SILCHENKO, op. cit., pp. 73-74.

35 Se si può parlare di "generazione di cibomaniaci", è pur vero che si tratta di una generazione consapevole dell'importanza di considerare insieme cultura alimentare e cultura dello sviluppo: DI VICO, Dario. Ipersalutisti e scettici a tavola. La generazione dei cibomaniaci. Corriere della Sera, 14/11/2016, pp. 22-23.

${ }^{36} \mathrm{Si}$ veda FOUCAULT, Michel. L'uso dei piaceri. Milano: Feltrinelli, 1984.

37 "La cultura della dieta e le sue varianti sono strutture culturali che abbiamo creato per provare a superare la nostra animalità. Attraverso le diete, l'uomo non solo mangia per restare in vita, ma entra a far parte di un edificio culturale più grande e più duraturo rispetto al suo corpo. È una sorta di rito dell'immortalità, e i riti vanno esplorati socialmente, mai in segreto.": ALLISON, Michelle. A dieta per essere immortali. Internazionale, n 1195, anno 24, pp. 90-92.

${ }^{38}$ SKRABANEK, Petr. The death of humane medicine and the rise of coercive healthism. Suffolk: The Social Affairs Unit, 1994, p. 39.
} 
diretto e duraturo per la sua materializzazione (letteralmente: incarnazione) e per il suo esercizio concreto.

\section{REFERÊNCIAS}

ALLISON, Michelle. A dieta per essere immortali. Internazionale, $\mathrm{n}^{\circ}$ 1195, anno 24, pp. 90-92.

Assemblea Generale delle Nazioni Unite. Dichiarazione Universale dei Diritti dell’Uomo. 1948.

BARALDI, Claudio; CORSI, Giancarlo; ESPOSITO, Elena. Luhmann in Glossario. I concetti fondamentali della teoria dei sistemi sociali. Milano: Franco Angeli, 2002.

BECK, Ulrich. Un mondo a rischio. Torino: Einaudi, 2003 (Das Schweigen der Wörter. Über Terror und Krieg. Frankfurt am Main: Suhrkamp, 2002).

BECK, Ulrich. La società del rischio. Roma: Carocci editore, 2000.

BECK, Ulrich. Dalla società industriale alla società del rischio. Questioni di sopravvivenza, struttura sociale e illuminismo ecologico. Teoria sociologica, 1, 1993, pp. 49-75 (Von der Industriellegesellschaft zur Risikogesellschaft: Überlebensfragen, Sozialstruktur und ökologische Aufklärung. In: KREMER, Will; A. KLEIN, Ansgar (Orgs.). Umbrüche der Industriegesellschaft: Herausforderungen für die politische Bildung, Vol. 284, Bundeszentrale für Politische Bildung. Bonn, 1990).

BECK, U.; BECK-GERNSHEIM, E. L'individualizzazione nelle società moderne. In: U. Beck; E. Beck-Gernsheim (Org.). I rischi della libertà. L'individuo nell'epoca della globalizzazione. Bologna: il Mulino, 2000, pp. 10-39 (Individualisierung in modernen Gesellschaften. Perspektiven und Kontroversen einer subjektorientierten Soziologie. In: Riskante Freiheiten. Individualisierung in modernen Gesellschaften. Frankfurt am Main: Suhrkamp, 1994).

BOURDIEU, Pierre; Saint-Martin Monique. Goftts de classe et styles de vie. In: Anatomie du goftt. Actes de la Recherche en Sciences Sociales, 5, out. 1976, pp. 18-43.

CEVOLINI, Alberto, Presentazione. In: LUHMANN, Niklas; CEVOLINI, Alberto (Org.). Il rischio dell'assicurazione contro i pericoli. Roma: Armando, 2013.

CORSI, Giancarlo. Introduzione. Malattia e salute: il contributo della teoria dei sistemi. In: Id. (Org.). Salute e malattia nella teoria dei sistemi. A partire da Niklas Luhmann. Franco Angeli, Milano, 2015.

DI VICO, Dario. Ipersalutisti e scettici a tavola. La generazione dei cibomaniaci. Corriere della Sera, 14/11/2016, pp. 22-23.

DOUGLAS, Mary. Il cibo come sistema di comunicazione. In: Antropologia e simbolismo. Religione, cibo e denaro nella vita sociale. Bologna: il Mulino, 1985. 
FOUCAULT, Michel. L'uso dei piaceri. Milano: Feltrinelli, 1984 (L'usage des plaisirs. Paris: Gallimard, 1984).

GIDDENS, Anthony, Le conseguenze della modernità. Bologna: il Mulino, 1994 (The Consequences of Modernity. Cambridge: Polity Press, 1990).

GOODY, Jack. Cooking, Cuisine, and Class. A Study in Comparative Sociology. Cambridge: Cambridge University Press, 1982.

LA CECLA, Franco. La pasta e la pizza. Bologna: il Mulino, 1998.

LONGO, Mariano. Strutture di società e semantica del soggetto. Lecce: Pensa Multimedia Editore, 2001.

LUHMANN, Niklas. Inflazione di pretese nel sistema delle malattie: una presa di posizione dal punto di vista della teoria della società (2015b). In: CORSI, Giancarlo (Org.). Salute e malattia nella teoria dei sistemi. A partire da Niklas Luhmann. Franco Angeli, Milano, 2015, pp. 52-70 (Anspruchsinflation im Krankheitssystem. Eine Stellungnahme aus gesellschaftstheoretischer Sicht. In: P. Herder-Dorneich, A. Schuller (Orgs.). Die Anspruchsspirale. Schicksal oder Systemdefekt?. Stuttgart: Kohlhammer, 1984).

LUHMANN, Niklas. Il codice della medicina (2015a). In: CORSI, Giancarlo (Org.). Salute e malattia nella teoria dei sistemi. A partire da Niklas Luhmann. Franco Angeli, Milano, 2015, pp. 40-51 (Der Medizinische Code. In: Soziologische Aufklärung 5: Konstruktivistische Perspektiven. Opladen: Westdeutscher Verlag, 1990, pp. 183-195).

LUHMANN, Niklas. CEVOLINI, Alberto (Org.). Il rischio dell'assicurazione contro i pericoli. Roma: Armando, 2013.

LUHMANN, Niklas. Globalization or World society: How to conceive of modern society? International Review of Sociology, Mar 1997b.

LUHMANN, Niklas. Die Gesellschaft der Gesellschaft. Frankfurt am Main: Suhrkamp, 1997a.

LUHMANN, Niklas. Society Shocked by Its Risks. Social Sciences Research Centre Occasional Paper, 17, 1996b.

LUHMANN, Niklas. Sociologia del rischio. Milano: Bruno Mondadori, 1996a (Soziologie des Risikos. Berlin: de Gruyter, 1991).

LUHMANN, Niklas. Individuo, Individualidad, Individualismo. Zona Abierta, 70-71, 1995, pp. 53-157 (Individuum, Individualität, Individualismus. In: Gesellschaftsstruktur und Semantik. Studien zur Wissenssoziologie der modernen Gesellschaft, vol. 3. Frankfurt am Main: Suhrkamp, 1989, pp. 149-259).

MARRONE, Gianfranco. Food meaning: From tasty to flavorful. Semiotica, 211, 2016, pp. 187201. 
MARRONE, Gianfranco. Senso e forma del cibo. Sulla semiotica dell'alimentazione. In: MONTANARI, Massimo (Org.). Il cibo nelle arti e nella cultura. Torino: Utet, 2015c, pp. 3-10.

MARRONE, Gianfranco. Food porn: 1'esposizione del cibo. In: MASSIDDA, Luca (Org.). EXPO 1851-2015. Storie e immagini delle Grandi Esposizioni. Torino: Utet, 2015b, pp. 3-6.

MARRONE, Gianfranco. La forma del latte. Allusioni erotiche ed emergenze del reale. Lexia. Rivista di semiotica, 19-20 giugno 2015a, pp. 331-346.

MARTINI, Sandra Regina. Construção do sistema social da saúde a partir da Teoria Sistêmica de Niklas Luhmann. Revista de Direito sanitario, 16, 1, 2015, pp. 112-127.

MARTINI, Sandra Regina. O sistema social da saúde: conceito, limites e possibilidades. Cad. Ibero-Amer. Dir. Sanit., 3, 1, (2014a), pp. 68-83.

MAYES, Christopher. Medicalization of eating and feeding. In: THOMPSON Paul B.; KAPLAN, David M. (Orgs.). Encyclopedia of food and agricultural ethics. Dordrecht: Springer, 2014, pp. 1-8.

SILCHENKO, Ksenia. Powered by Health: Healthism in Food Marketing and Consumer Research. A Systematic Revier and a Critical Disourse Analysis. 2017. Tese (Doutorado) Università degli Studi di Macerata, 2017.

SKRABANEK, Petr. The death of humane medicine and the rise of coercive healthism. Suffolk: The Social Affairs Unit, 1994.

SPENCER-BROWN, George. Laws of form. Bohmeier Verlag: Leipzig, 2014 (1969).

WHO. Constitution of the World Health Organization. 1946. 